NBER WORKING PAPER SERIES

\title{
INTER-TEMPORAL R\&D AND CAPITAL INVESTMENT PORTFOLIOS FOR THE ELECTRICITY INDUSTRY'S LOW CARBON FUTURE
}

\author{
Nidhi R. Santen \\ Mort D. Webster \\ David Popp \\ Ignacio Pérez-Arriaga \\ Working Paper 20783 \\ http://www.nber.org/papers/w20783 \\ NATIONAL BUREAU OF ECONOMIC RESEARCH \\ 1050 Massachusetts Avenue \\ Cambridge, MA 02138 \\ December 2014
}

We wish to thank Jake Whitcomb for his excellent research assistance in helping prepare the manuscript for publication. We also thank Andres Ramos, Javier Garcia, and Joseph Sussman for valuable discussions on electricity generation expansion planning and energy innovation, and two anonymous reviewers. Finally, we gratefully acknowledge financial support from U.S. National Science Foundation Grant Nos. 0825915 and 0835414, and the MIT Science and Technology Initiatives Spain Program, Martin Family Sustainability Fellows Program, and Office of the Graduate Dean for Education John S. Hennessy Fellowship Program. The views expressed herein are those of the authors and do not necessarily reflect the views of the National Bureau of Economic Research.

NBER working papers are circulated for discussion and comment purposes. They have not been peerreviewed or been subject to the review by the NBER Board of Directors that accompanies official NBER publications.

(C) 2014 by Nidhi R. Santen, Mort D. Webster, David Popp, and Ignacio Pérez-Arriaga. All rights reserved. Short sections of text, not to exceed two paragraphs, may be quoted without explicit permission provided that full credit, including (C) notice, is given to the source. 
Inter-temporal R\&D and Capital Investment Portfolios for the Electricity Industry's Low Carbon Future

Nidhi R. Santen, Mort D. Webster, David Popp, and Ignacio Pérez-Arriaga

NBER Working Paper No. 20783

December 2014

JEL No. Q40,Q42,Q55

\begin{abstract}
This paper explores cost-effective low-carbon R\&D and capital investment portfolios for the electricity generation sector through 2060. We present a novel method for long-term planning by combining an economic model of endogenous non-linear technical change and a generation capacity planning model with key features of the electricity system. The model captures the complementary nature of technologies in the power sector; physical integration constraints; and the opportunity to build new knowledge capital as a non-linear function of R\&D and accumulated knowledge, which reflects the diminishing marginal returns to research characteristic of the energy innovation process. We show portfolios for future scenarios with and without carbon emission limits, and demonstrate the importance of including various features by comparing results from a reference version of the model to results from alternative versions that omit these features. Our results caution that using economic frameworks that do not incorporate critical electricity and innovation system features may over- or under-estimate the value of emerging technologies, and therefore the cost-effectiveness of R\&D opportunities.
\end{abstract}

Nidhi R. Santen

Belfer Center for Science and International Affair

Harvard Kennedy School

79 John F Kennedy Street

Mailbox 53

Cambridge, MA 02138

nidhi_santen@hks.harvard.edu

Mort D. Webster

Department of Energy and Mineral Engineering

Pennsylvania State University

123 Hosler

State College, PA 16802

mort@psu.edu
David Popp

Department of Public Administration

and International Affairs

Syracuse University

The Maxwell School

426 Eggers Hall

Syracuse, NY 13244-1020

and NBER

dcpopp@maxwell.syr.edu

Ignacio Pérez-Arriaga

Instituto de Investigacion Tecnologica (IIT)

Universidad Pontificia Comillas, Alberto Aguilera, 28015 Madrid, Spain

ignacio.perez@iit.upcomillas.es 


\section{Introduction}

Effectively managing carbon dioxide $\left(\mathrm{CO}_{2}\right)$ emissions from fossil-based electric power generation is critical for executing a comprehensive global climate change mitigation and sustainable energy plan. In the United States, for example, the electric power generation sector is responsible for approximately forty percent of the country's annual emissions (EIA 2012). Unfortunately, many technologies for significantly reducing $\mathrm{CO}_{2}$ emissions from the electricity sector are either in early conceptual stages or are only available at relatively high costs or small scales, requiring additional research and development (R\&D). Meanwhile, the industry continues to meet increasing electricity demands with carbon-emitting technologies that are both commercially available and economically viable. To address the dilemma of resolving increasing electricity demands with emission reduction goals, it would be useful to find costeffective investment portfolios for R\&D on emerging electricity technologies and new generation capacity at the utility or power producer level (DOE 2011). On one hand, incentivizing existing low-carbon technology adoption can play an important role in nearterm carbon reductions. On the other hand, supporting R\&D can benefit long-term carbon reductions by commercializing new, less expensive low-carbon technologies for the future. What is the right balance between, and timing for, these two efforts?

Previous studies of optimal low-carbon R\&D and capital investment portfolios for the electric power generation sector have neglected one of two critical aspects of the electricity industry and the energy R\&D process needed to provide useful insight. First, existing research in this area lacks resolution of the critical structure of, and engineering constraints within, the electric power system. The necessary cooperation between highly 
complex, time-dependent technical operations and the infrastructure itself sets electricity apart from other large-scale, commodity-delivering infrastructure systems. This characteristic requires a diverse portfolio of generating technologies during any given period of time in the system complementing each other to deliver reliable electricity. Research is underway to integrate detailed system-level features into economic modeling frameworks (e.g., Tapia-Ahumada, Octaviano, Rausch, \& Pérez-Arriaga 2014; Shawhan et al. 2014), but the typical approach for studying the joint energy R\&D and capacity planning problem thus far still uses a "top-down" economic perspective. This approach lacks explicit disaggregated capital, variable, and other fixed costs; real operational constraints (e.g., solar and wind intermittency, nuclear plant cycling); and adequate temporal resolution in the demand-supply power balance over the course of a year (e.g., Goulder \& Schneider 1999; Goulder \& Mathai 2000; van der Zwaan, Gerlagh, Klaassen, \& Schrattenholzer 2002; Buonanno, Carraro, \& Galeotti 2003; Popp 2004; Popp 2006; Baker \& Solak 2013; Pugh et al. 2011).

Second, the studies that do represent the power system in detail typically omit an explicit endogenous representation of the energy R\&D process. Due to data limitations, most existing studies for electricity investment portfolio planning rely on exogenous discrete time trends or experience curves to represent the change in costs of different technologies over time, neglecting the role of R\&D (e.g., Short et al. 2011; Ross 2008; Grubb, Kohler, and Anderson 2002; Messner 1997; Mattheson \& Wene 1997; Loulou, Goldstein, \& Noble 2004; EIA 2009; Seebregts et al. 1999; Morris 2002; Berglund \& Soderholm 2006; Kypreos \& Barreto 2000). A few studies have included R\&D, but assume linear (or one-to-one) relationships between $\mathrm{R} \& \mathrm{D}$ investments and new technical 
knowledge creation (e.g., Kouvaritakis, Soria, \& Isoard 2000; Turton \& Barreto 2004; Barreto \& Kypreos 2004; Miketa \& Schrattenhozler 2004). The linear approach does not capture diminishing marginal returns to research, for which there is considerable empirical evidence and theoretical support. The functional form of the returns to R\&D is a critical assumption, because empirical studies suggest that the state of scientific knowledge significantly affects the overall outcome of the R\&D investment at a given point in time (Popp, Newell, \& Jaffe 2010; Popp 2002). There have been a few studies that represent both rich technological details of the power system alongside key features of the innovation process using a "hybrid" perspective (e.g., Mann, Mendelsohn, \& Richels 1995; Bosetti, Carraro, Galeotti, Massetti, \& Tavoni 2006). For example, Bosetti et al. (2006) assume a non-linear functional form for returns to $R \& D$ that exhibits diminishing returns. However, this study and other similar hybrid modeling efforts still use a relatively coarse resolution of the power sector. Although hybrid modeling efforts offer the promise of improved models in the future, many current economic models omit the detailed engineering cost structures of the different generation technologies, the temporal variability of electricity demand, and the interaction between different technologies with demand to determine optimal operations.

In this paper, we explore socially optimal low-carbon R\&D and capital investment portfolios for the electric power generation sector, using a model with endogenous non-linear R\&D and a relatively detailed representation of the power system ${ }^{\mathrm{i}}$. We present a novel modeling approach that considers the complementary roles that generation technologies play within the power system, the physical integration

\footnotetext{
${ }^{\mathrm{i}}$ We refer to the "optimal" investment strategy here, and throughout the remainder of the paper, in reference to our numerical implementation of the investment problem rather than to an "absolute" optimum.
} 
constraints they face, and the economics at play in electric utilities' least-cost investment decisions. Our approach also considers specific characteristics of the innovation process, including the opportunity to build knowledge capital as a non-linear function of R\&D investments and the current state of technical knowledge, and explicitly represents diminishing marginal returns to research inherent in the energy innovation process. Note that in each respective area-electric power generation capacity investment planning and energy R\&D investment planning - research and modeling capability has steadily progressed. For example, state-of-the-art "bottom-up" electricity generation capacity planning models integrate hourly, or even more frequent, temporal demand; unit-level features such as start-ups and shut-downs; geographic heterogeneity with respect to technology base and demand; and/or very sophisticated representations of intermittent renewables such as wind and solar (e.g., Blanford et al. 2014; Palmintier 2013; Short et al. 2011). Our goal is to demonstrate the value for energy planning of combining an engineering cost-based structure with an economic model of the innovation process. Doing so requires bringing together some of the most salient features of power system operations and the energy innovation process into a single modeling framework. However, we intentionally keep the approach stylized, and avoid integrating many additional state-of-the-art modeling capabilities, to keep the results as straightforward and interpretable as possible.

Overall, the model indicates that the optimal R\&D strategy should focus on renewable energy technologies in the near- and medium-term, with gradual and increasing R\&D investment in nuclear power in the longer-term to meet carbon limits. The optimal capital investment strategy follows a similar pattern, with the exception that 
under carbon limits, investment in nuclear power is deferred until costs are sufficiently reduced from R\&D. We demonstrate that when realistic details of the power system are represented, the optimal strategy is qualitatively different from the resulting strategies from current state-of-the-art methods. Specifically, the improved model shows that large R\&D and capital investments in baseload technologies such as coal with carbon capture and sequestration (CCS) may not be optimal, given the impracticality for nuclear plants to cycle (i.e., frequent startups and shutdowns), and the respective cost structures and innovation potentials of these technologies. It also shows that existing modeling approaches may underestimate the contribution of variable renewable technologies, such as solar, to the power system, and thus the cost-effectiveness of their R\&D opportunities. Finally, the explicit introduction of diminishing marginal returns to R\&D cautions against underinvesting in emerging technologies such as renewable energy and nuclear, for which it is necessary to support on-going technological change and associated costreductions.

The remainder of this paper is organized as follows. The next section formulates the specific investment problem we seek to solve, and provides details about the new modeling approach, describing its structure and calibration. In Section 3, we use the new modeling approach to study optimal $R \& D$ and capital investment portfolios with and without carbon limits. We also solve for the optimal investment strategy for two additional cases-one without critical power system details and another without diminishing returns to $\mathrm{R} \& \mathrm{D}$ - in order to demonstrate the value of explicitly representing these components of the problem. Finally, we show the sensitivity of the optimal investment strategy to key innovation process characteristics. The new modeling 
approach allows explicit study of the effects of different components of the knowledge building process on optimal $R \& D$ and capital investments, which we find valuable given recent empirical evidence showing notable variation between technology groups in the contribution of their knowledge stocks on successful innovation (Popp, Santen, FisherVanden, \& Webster 2013). Section 4 provides a discussion of the results and suggests future research directions.

\section{Modeling framework}

We begin with a traditional engineering cost-based electricity generation capacity expansion optimization model (e.g., Turvey \& Andersen 1997; Hobbs 1995) with ten technology categories, and modify it to simultaneously choose R\&D investments for four emerging low-carbon technologies: coal with carbon capture and sequestration, nuclear, wind, and solar. The cost-based structure of the model includes technology specific costs; temporal load variability; and engineering constraints governing the power balance, supply reliability, intermittent wind and solar power, and constraints on cycling nuclear generators. We build upon previous modeling work that incorporates both endogenous experience curves and endogenous $R \& D$ dynamics to allow overnight capital costs to evolve as a function of capital investments and technological innovation (e.g., Barreto \& Kypreos 2004; Fischer \& Newell 2008). However, the model of the innovation process exhibits diminishing returns with increasing research effort (e.g., Popp 2004; Popp 2006; Bosetti et al. 2006). The planning horizon is sixty years, with capital investment and R\&D investment decisions made every five years. A centralized planning approach is used, which assumes that a central decision maker simultaneously 
makes national investment and generation decisions. This centralized approach is appropriate for the long-range strategic policy and technology-planning objective of the current question, and the large structural uncertainties inherent in short-term market behaviors of individual firms over such long time scales (Pérez-Arriaga \& Meseguer 1997). Below, we present the key features of the model, data, calibration, and solution approach below. Full model details are provided in the appendix.

\subsection{Objective}

Every five years, the decision maker chooses new power plant capacities, $N C_{g, t}$, for each technology $g$, and $\mathrm{R} \& \mathrm{D}$ investments, $R D_{g^{*}, t}$, for the subset of emerging technology categories $g^{*}$, to minimize the net present value of total system costs,

$$
\min _{N C_{g, t}, R D_{g^{*}, t}}\left[F C_{g, t}+V C_{g, t}+R D_{g^{*}, t}\right](1-r)^{-t}
$$

where $g^{*} \in g, F C_{g, t}$ represents the total fixed costs (overnight capital and fixed O\&M costs) of technology category $g$ in period $t, V C_{g, t,}$ represents the total variable costs (fuel and variable O\&M costs) of technology $g$ in period $t$, and $r$ is the discount rate.

In addition to the constraints that define the design and operation of the underlying electric power system, summarized below, the key constraint driving the optimal investment strategy is a cumulative carbon emissions cap, ecap, applied to the power sector, 
$\sum_{t}^{T} E_{t} \leq e c a p$

where $E_{t}$ represents total carbon emissions from the electricity sector during each period. The existence of a cumulative cap forces a choice between reducing emissions now versus later, given current technology costs and future R\&D-based cost-reduction potential.

\subsection{Electricity system operations}

We parameterize the power system model to be qualitatively representative of the U.S. generation portfolio and electricity demand (EIA 2011; Short et al. 2011). Table 1 lists the technology categories and associated values used for key parameters in the generation expansion problem. Electricity demand is characterized using an annual load duration curve with seventeen time slices representing four seasons, four daily segments, and a "super peak" representing the highest forty (non-consecutive) hours of demand. Figure 1 shows the duration and power level associated with each segment of the load duration curve.

Key constraints of the traditional power generation expansion planning and optimal dispatch problem are retained, including those dictating energy demand balance, reliability of supply, and energy resource availabilities. To this, we introduce several additional details. First, we model to first order the effects of intermittent renewable resources and the dominant operational constraints of nuclear power plants. Using a netload approach, both solar power and nuclear power are defined as "non-dispatchable," and all other technologies compete on cost in order to meet demand. Wind is modeled as 
"dispatchable" in order to allow its curtailment. Solar power is also represented as operating only during demand slices that correspond to the daytime. ${ }^{\text {ii }}$ Second, a high retirement rate is used for old coal and old oil/gas steam plants, as a very large capacity of these technologies is expected to retire in the U.S. over the next one to two decades. A high retirement rate for nuclear power is also included, given the aging stock of the U.S. nuclear fleet. Conversely, new investments in old coal technology, old oil/gas steam, and hydropower are not allowed in the model because these technologies are outdated or near their energy resource limits in the U.S. Third, we represent the inability to scale up emerging technologies rapidly and without limit by assuming a constraint on the rate of change of installed capacities between periods. Table 3 shows values assumed for additional parameters associated with the problem as defined.

\subsection{Technical change dynamics}

The process of innovation enters the model through two distinct complementary pathways. Building on the recent empirical and numerical modeling literature, we use a two-factor learning curve (2FLC) to simultaneously represent learning-by-doing (LBD) and learning-by-searching (LBS) (Klaassen, Miketa, Larsen, \& Sundqvist 2005; Soderholm \& Klaassen 2007; Miketa \& Schrattenholzer 2004; Barreto \& Kypreos 2004). Through the 2FLCs, the overnight capital cost of a technology is decreasing in the technical knowledge stock for the technology, and decreasing in the cumulative capacity installed. While the 2FLC formulation applies to the emerging technology groups (coal

\footnotetext{
ii Representing each of these constraints can be approached in many ways, with varying degrees of sophistication. Our stylized model includes the minimum degree of realism we believe is necessary to study whether results change after integrating power system operation and innovation process details.
} 
with CCS, nuclear, wind, and solar), all technologies for which new capacity can be added continue to learn via LBD via a traditional one-factor learning curve $\mathrm{e}^{\mathrm{iii}}$.

The technical change dynamics in the model are summarized with the following three equations:

$$
\begin{aligned}
& N E W K_{g, t}=\alpha_{g} R D_{g, t}^{\beta} K S_{g, t}^{\phi} \\
& C_{g, t}=\frac{C_{g, 0}}{\left(I C_{g, t}^{\eta 1 g}\right)\left(K S_{g, t}^{\eta 2 g}\right)} \\
& K S_{g, t+1}=N E W K_{g, t}+\delta_{g} K S_{g, t}
\end{aligned}
$$

Equation (3) represents the production of new knowledge, $N E W K_{g, t}$, for technology $g$ in time period $t$, defining it as a function of $\mathrm{R} \& \mathrm{D}$ investment, $R D_{g, t}$, and the cumulative technical knowledge stock, $K S_{g, t}$, for technology $g$ in time period $t$. The parameter $\beta_{g}$ represents the contribution of $\mathrm{R} \& \mathrm{D}$ dollars invested in the creation of new knowledge, $\varphi_{g}$ represents the contribution of the current knowledge stock in the creation of new knowledge, and $\alpha_{g}$ is a technology-specific scalar used to calibrate the behavior of the new innovation possibilities frontier to the current learning literature. Diminishing

\footnotetext{
iii There are many other pathways for technical change, including but not limited to spillovers, learning-byassociation, and exogenous technical change (progress of the overall economy). Additionally, there are many outstanding theoretical and empirical issues with respect to definition and measurement (e.g., Clarke, Weyent, Birky 2006; Nordhaus 2013). Integrating the full range of these is beyond the scope of this paper. Instead, we keep the model stylized including learning-by-doing and learning-by-researching as the pathways most directly connected to decisions about new capacity and R\&D.
} 
returns to research are incorporated in this "innovation possibilities frontier (IPF)," by constraining the sum of $\beta_{g}$, and $\varphi_{g}$ to less than 1.0 .

Equation (4) represents the two-factor learning curve that combines LBD and LBS. $C_{g, t}$ is the capital cost of technology $g$ in time period $t, C_{0, g}$ is the initial capital cost at unit capacity and knowledge stock, $I C_{g, t}$ is the cumulative installed capacity in $\mathrm{GW}$ of technology $g$ in time period $t, K S_{g, t}$ is once again the cumulative human knowledge stock for technology $g$ in time period $t$, and $\eta 1_{g}$ and $\eta 2_{g}$ are the learning-by-doing and learningby-research output elasticities for technology $g$, respectively. As indicated by the subscript $g$, the parameters $\eta 1_{g}$ and $\eta 2_{g}$ are technology specific; their interpretation follows directly from traditional experience curve "progress-ratio" calculations where 1 $2^{\eta i}$ describes the cost reduction that occurs from a doubling of capital stock $(\eta 1)$ or knowledge stock $(\eta 2)$ (e.g., Ibenholt 2002). For the non-emerging technology groups, $\eta 2_{g}$ is set to zero, resulting in one factor LBD curves being applied to the evolving overnight capital costs $^{\text {iv }}$. Technology specific parameters of the IPF and 2FLC, listed in Table 4, are calibrated to estimates in the published literature ${ }^{\mathrm{v}}$. Specifically, the scaling parameter, $\alpha_{g}$, minimizes the sum of squared differences between capital cost reductions as formulated here, and capital cost reductions based on published two-factor LBD and LBS rates, and R\&D and knowledge stock output elasticities (Barreto \& Kypreos 2004; Popp 2006).

\footnotetext{
iv For consistency, one-factor LBD parameter values were re-constructed from the 2FLC values employed by Barreto \& Kypreos (2004), based on achieving the same cost-reduction over capital stocks.

$\mathrm{v}$ There is a wide range of estimates in the published literature on learning rates, as well as considerable debate about the appropriateness of using historical data to estimate future costs for emerging technologies. Addressing these issues is beyond the scope of this paper, which aims to present a new framework, but it is important for the reader to note that these differences exist and could impact the specific numerical results presented here.
} 
Equation (5) represents the knowledge building process, capturing the stock nature of the technical knowledge capital dimension in this problem. The parameter $k_{g t}$ represents the new knowledge gained for technology $g$ during period $t$ through the innovation possibilities frontier, and $\delta_{g}$ represents a technology-specific decay rate for the knowledge stock from one period to the next.

\section{$2.4 \quad$ Numerical Implementation}

We solve the electricity generation capital and R\&D investment planning problem numerically, as a non-linear program. We use the GAMS modeling environment, and a standard non-linear programming (NLP) solver, CONOPT. A 20-point seeding algorithm is also used to set initial values widely distributed over the solution space. The optimum is the solution to the NLP that minimizes total system costs across all twenty seeds. We address terminal conditions for this multi-period decision problem by running the 60 -year planning problem for an additional 40 years. Results from only the first 12 (5-year) periods are used in each of the analyses to ensure that decisions being made at or near the end of the planning horizon are not "end-of-world" artifacts or a result of artificially imposed terminal conditions. 


\section{Results}

In this section, we present the results of four numerical modeling experiments. ${ }^{\mathrm{vi}}$ We first present the optimal investment strategy resulting from solving the reference model with no carbon limit and then with a moderately stringent carbon limit equivalent to reducing business-as-usual carbon emissions from the power generation sector by fifty percent annually. Next, we show the impact of modeling critical power system details and diminishing returns to $\mathrm{R} \& \mathrm{D}$ on the resulting optimal strategy. Our focus is to explicitly show how the optimal investment strategy changes when these features are considered, and to demonstrate the value for energy planning of combining an engineering cost-based structure with an economic model of the innovation process. Finally, we present results from a sensitivity analysis of the optimal investment strategy on the efficiency of R\&D, and on the strength of the technology knowledge stocks. An additional advantage of the modeling framework we present is the capability to directly study component drivers of new technology knowledge creation. The sensitivity analysis results illustrate the behavior of the model with respect to these components.

\subsection{Reference optimal investment strategy}

When carbon emissions from the power generation sector are allowed to grow unconstrained, the new modeling framework with endogenous R\&D-based technical change and diminishing returns shows that it is optimal in the near-term to invest aggressively in wind power R\&D, and to a lesser extent in solar PV R\&D, and then to

\footnotetext{
${ }^{\mathrm{vi}}$ Note that throughout this paper we refer to investment strategies using the names of the technologies we have included in our model, but in reality the results are driven by the underlying engineering and cost characteristics of the technologies themselves. Therefore, the reader should feel free to consider the strategies more generically, using the technology characteristics we have included as a benchmark.
} 
decrease investments of both to negligible amounts after two to four decades (Figure 2). The temporal pattern of optimal capital investments (deployment) for the reference model (Figure 3) helps to explain this behavior. Several gigawatts of wind are installed in the second and third periods in order to make up a sizeable share of the total installed capacity by the fourth period. R\&D investments reflect this deployment pattern. Investments occur early and aggressively to reduce costs as much as possible before massive deployments. Solar PV deployments in the fifth through the ninth periods occur on a much smaller scale, but solar R\&D investments similarly reflect the capacity increases over time. Investments begin early in order to reduce capital costs as much as possible, but to a lesser extent than for wind, and they occur over a longer interval of time because deployments occur further in the future.

Note that wind and solar PV technology R\&D and deployments appear even in the absence of a carbon emissions limit; this is due to their specific cost structures and the presence of learning in the model. Wind technology is already close to being costcompetitive with the other technologies' capital costs (See Table 1). Because of the competitive cost and relatively rapid learning rates, near-term R\&D investments and deployment in wind technology yields significant cost savings during future installations, which in turn encourages further deployment ${ }^{\text {vii, viii }}$. Solar PV technology, despite

\footnotetext{
${ }^{\text {vii }}$ When engineering details of power system operations and merit-order effects are considered in combination with endogenous learning opportunities, as in the current modeling framework, it is common to witness significant wind technology deployment; fuel costs are zero, other variable costs are among the lowest in the technology suite, and overnight capital costs become quite low due to cumulative LBD, LBS, and other effects). In our results, the amount of wind (or any other technology) R\&D and deployment can also be attributed to previous technology support policies such as a production tax credit or state RPS since implicit in the learning rates and thus costs is the effect of past installations motivated by these policies. Note, however, that technology capital costs do not directly reflect the impact of such support policies.

${ }^{\text {viii }}$ While capital and other engineering costs for wind power decrease to the point of being costcompetitive with some conventional technologies, note that other constraints continue to exist (e.g., transmission capacity) that can prevent actual wind deployment and are not represented in our model.
} 
significantly higher capital costs compared with other technologies, is also developed and deployed due to its high learning potential (both through experience and R\&D) (See Table 3). In contrast, low-carbon coal with CCS and zero-carbon nuclear, both technologies with high relative overnight capital costs but lower learning potentials, do not have sufficient incentive for either R\&D or deployment in the absence of a carbon constraint. Figure 2 also shows the relative allocation of the $R \& D$ amount for the optimal investment strategy in each period, and indicates the substantial early R\&D investment in wind technology, followed by a delayed, but prolonged investment in solar PV. For the remainder of the paper, we will present the optimal R\&D investment strategy in terms of the ratio of $R \& D$ by technology to the total $R \& D$, and emphasize insights into the relative investment allocation, rather than the absolute magnitudes from our stylized model.

Under a moderately stringent carbon limit, one that is equivalent to constraining annual carbon emissions from the power generation sector to fifty percent of 2010 "business-as-usual" emissions, the optimal strategy involves R\&D and capital investments in nuclear technology, in addition to the same absolute wind and solar development and deployment plan as with no carbon limit. Nuclear technology R\&D investments occur more gradually than wind and solar, peaking after 35 years, just before new nuclear capital investments occur (Figures 2 and 3). Because nuclear generation is baseload (operating for most hours of the year), emits no carbon emissions, and has lower capital and operating costs than coal with CCS - the alternative baseload low carbon technology - it plays an important role in reducing cumulative carbon emissions. Note the significant amount of electricity generated by nuclear in Figure 3 under a carbon limit. However, the deployment of nuclear is deferred until later periods to take 
advantage of the capital cost reductions from early R\&D investments. Under the carbon limit, nuclear technology has the largest share of R\&D (Figure 2). We note that neither development nor deployment of coal with CCS occurs in the optimal strategy for the reference model. This is a result of its higher overnight capital costs and fuel costs relative to alternative low-carbon technologies (Table 1), combined with its relatively lower learning rates (Table 3). ${ }^{\text {ix }}$

\subsection{The effect of power system details}

The results described above have been obtained using an engineering cost-based structure for the electric power R\&D and capital investment planning model instead of a typical economic modeling structure, to ensure that critical physical and technological characteristics of the power system are represented in sufficient detail. In this section, we present results obtained with an alternative formulation of the model (hereafter referred to as the "simplified power system model") with several important engineering constraints omitted to show that the optimal investment strategy is indeed different than that in the reference model. Specifically, we neglect the explicit representations of temporal load variability, time-of-day output constraints for solar PV panels, and the inability of nuclear plants to cost-effectively manage repeated start-up and shut-downs. Instead, we assume an average annual demand value, a solar PV panel output that assumes a specific average capacity factor $(30 \%)$, and neglect the baseload "must-run" constraint we place on nuclear power generation. These assumptions are consistent with the vast majority of

\footnotetext{
ix The numerical model considers the possibility that coal with CCS and nuclear power technologies can incur an upper bound in deployment for political or physical reasons (e.g., concerns about nuclear waste disposal or public safety, physical limitation of carbon sequestration sites) by including a maximum installed capacity of $30 \%$ (for each) of total system capacity. However, neither constraint is active in the solution, indicating that the results are robust to these types of concerns within this limit.
} 
economic energy models that focus on the rest of the economy but use a simplified electricity sector with an annual demand representation.

Figures 4 and 5 present the optimal R\&D and capacity investments, respectively, resulting from the simplified power system model. Without a carbon limit, the optimal R\&D investment strategy focuses solely on wind technology; R\&D investments in solar PV are not part of the investment strategy, which differs significantly from the results obtained with the reference model. Solar PV technology is also dropped from the deployment plan, with wind making up the balance. Furthermore, under a carbon limit, R\&D and capital investments in solar are effectively zero. In contrast to the reference model, under the carbon limit wind R\&D investments continue throughout the sixty-year planning horizon (albeit at smaller share of total R\&D relative to nuclear technology). Another significant difference in this version is that the optimal investment strategy includes both baseload low-carbon technologies, nuclear and coal with CCS. Investment in nuclear R\&D is still optimal under the alternate model, and remains at a relatively high share throughout the planning horizon (rather than starting out small and gradually increasing over time). Coal with CCS generation changes most dramatically under this simplified power system formulation, and exhibits positive optimal R\&D and capital investments throughout the planning horizon, in contrast to zero investment in the reference model. In fact, investment is so high for coal with CCS that by the seventh period it makes up the largest share of $R \& D$ expenditures across the four emerging technologies.

Why are R\&D investments into coal with CCS justified under a carbon limit in the alternate model? A main reason is the absence of the must-run constraint on nuclear 
generation. Without that constraint, both coal with CCS and nuclear technology can contribute to meet electricity demand. Nuclear generation, initially with lower overnight capital cost, is deployed sooner in this version than in the reference model, and therefore also has high R\&D investment shares in early periods. Coal with CCS is initially higher cost, and is consequently deployed in later periods, after $R \& D$ investments have had sufficient time to reduce capital costs and increase its competitiveness. The R\&D investment share in coal with CCS starts off small in the early periods, and then rises swiftly over the next few periods. The optimal deployment strategy under the simplified power system formulation also includes less wind and more natural gas plants.

The reason why the optimal R\&D investment plan from the simplified power system model does not include solar PV is that the model does not differentiate between the times when solar generation is and is not available. Solar generation is effectively assumed to be available at any time of day or in any season regardless of solar incidence, but only and always at 30\% output, as opposed to the much higher levels for some seasons and times of day. A maximum capacity factor of $30 \%$ combined with its high initial capital cost, makes this technology not cost-effective. In general, we caution that using economic modeling frameworks that do not resolve temporal variation may underestimate the value of variable generation technologies such as solar PV, and therefore may also underestimate cost-effective R\&D opportunities. Similarly, for baseload technologies with specific engineering constraints such as nuclear, such frameworks may overestimate the opportunity for competing baseload technologies (e.g., coal with CCS, gas) and the corresponding value of $R \& D$ investment into these technologies. 


\subsection{The effect of diminishing returns to research}

An important contribution of this work is the incorporation of diminishing returns to research into an R\&D and capital investment planning model for the power sector. Here, we demonstrate the value of taking this realistic approach, relative to the common approach that assumes linear R\&D-knowledge building relationships without diminishing returns. We formulate a third version of the model, which replicates the reference version from Section 3.1 with the important exception of the mechanism through which knowledge increases about emerging technologies. Specifically, in this version, we replace Equation (3) in the reference model with:

$k_{g, t}=R D_{g, t}$,

where new knowledge, $k$, for technology, $g$, in time, $t$, is a one-to-one function of the dollars invested in technology, $g$, and time, $t$. This formulation closely matches the dominant assumption in many energy models, which equates R\&D dollars with new knowledge. We refer to this version as the "linear R\&D" model version.

Figure 6 shows the optimal R\&D investment strategies with and without a carbon limit when the linear knowledge production function is assumed, omitting diminishing returns. In the absence of a carbon limit, the relative shares of R\&D investments into wind and solar technologies in the early periods is approximately the same, but $R \& D$ into solar ceases after the fifth period. The same pattern holds for wind and solar R\&D investment under a carbon limit. In the linear R\&D model, R\&D into nuclear technology 
under the carbon limit has a higher optimal share in the first two periods, after which investment stops abruptly. In contrast, when diminishing returns are considered (Figure 2), nuclear R\&D begins gradually, and becomes dominant for most of the planning horizon.

In general, the impact of ignoring the decreasing returns from research effort as a function of cumulative effort is to overestimate the effect of $R \& D$ into technologies. The linear R\&D model also results in a different temporal path of optimal R\&D investments, leading to a "boom-and-bust" pattern of investing a lot in early periods, after which R\&D ceases. This is particularly true for technologies such as solar PV and nuclear, which have delayed deployment and thus investment in the reference model spread out over many periods. For technologies with low learning-by-searching rates but large deployments in the reference model (e.g., nuclear under a carbon limit), the linear R\&D model chooses a disproportionate level of R\&D effort in the early periods. The shift in the timing of R\&D, in turn, changes the optimal capital investment pattern, resulting in earlier deployment of nuclear generation (Figure 7), displacing combined cycle natural gas plants during those periods. A similar pattern is seen for nuclear technology R\&D investments under a carbon constraint in the engineering cost model of Barreto \& Kypreos (2004), which assumes linear R\&D-based knowledge building and the same low learning rate. In their model, technologies with higher learning rates witness continued R\&D investment over their planning horizon. 


\subsection{Optimal investment strategies v. knowledge building components}

An additional benefit of representing endogenous learning-by-searching with an innovation possibilities frontier (IPF), such as Equation (3) of the reference model, is the ability to explicitly study the impact of assumptions about R\&D efficiency (parameter $\beta$ ) and about the effect of the knowledge stock level in generating new knowledge (parameter $\varphi$ ) on the inter-temporal knowledge building process, and consequently on the optimal investment strategy. This is particularly salient given evidence in the technical change literature showing variation across energy technologies in the contribution of their knowledge stocks to successful innovation (Popp et al., 2013). In the reference model (Section 3.1), we assume values of $\beta$ and $\varphi$ from the literature, assigning all emerging technologies the same values ${ }^{\mathrm{x}}$. Here we summarize results from sensitivity analyses of the optimal strategy to both of these characteristics of the innovation process. We focus on the impact on $R \& D$ investments, because capacity investments are less sensitive to changes in the IPF parameters in our model.

Figure 8 shows the share of $R \& D$ investments, defined by the peak investment over time, in each of the four emerging technology groups for a range of $R \& D$ investment efficiency (parameter $\beta$ ) values under a carbon limit (reference value for $\beta$ is 0.1). With increasing R\&D efficiency, the shares of wind and solar power (technologies with high learning rates and early R\&D) investments display only mild sensitivity; the R\&D investment shares for these technologies only decrease slightly as R\&D efficiency

\footnotetext{
${ }^{\mathrm{x}}$ Although parameters $\beta$ and $\varphi$ of the innovation possibilities frontier are the same across the four emerging technologies, a scaling parameter $\alpha$ is used to calibrate each technology to technology specific learning values in the literature allowing each technology to follow its own cost-reductions over R\&D investment (See Section 3 and supplemental information for more details).
} 
increases. In contrast, nuclear power, which has a much lower learning rate and an optimal path of $R \& D$ that peaks in later periods, experiences increasing investment shares with increasing R\&D efficiency. The general trend is robust to R\&D investment efficiencies, however; it is optimal to invest the largest share into nuclear, the next largest share into wind, the least into solar, and none into coal with CCS.

The optimal investment pattern is more sensitive to assumptions about $\varphi$, the elasticity of new knowledge to current knowledge stock levels (Figure 9). As the impact of knowledge stock on new knowledge increases, the relative share of wind R\&D investments increase and the relative share of nuclear R\&D investments decrease. Specifically, for values of $\varphi$ below approximately 0.4 (reference value is 0.54 ), it is optimal to invest the majority of R\&D into wind over nuclear, but for values greater than 0.4 , it is optimal to invest the majority of $R \& D$ into nuclear power. The effect on optimal R\&D share into solar and coal with CCS is more robust to the knowledge stock effect. It is optimal to spend approximately one-tenth of total R\&D expenditures on solar PV technology for any value of knowledge stock strength. The optimal strategy continues to exclude R\&D or capital investments into coal with CCS across all values of $\beta$ and $\varphi$ studied here.

We conclude that for technologies for which early R\&D investments are optimal and learning rates are high (e.g., wind), more favorable innovation processes shift the optimal strategy away from R\&D investment. The intuition is that if the same amount of R\&D expenditures yields greater returns, there is no need to spend extra money. Additionally, because expenditures take place in the first few years for these 
technologies, there is less time to benefit from accumulating knowledge. However, for technologies with low learning rates and deferred R\&D investments (e.g., nuclear), increasing the strength with which the knowledge stock contributes to new knowledge (and ultimately cost reductions) incentivizes increasing R\&D investments. In these cases, there is more time for benefits from additional R\&D to accrue. Discounting of future period $R \& D$ investments also makes an increase in $R \& D$ not as costly a decision.

Overall, when compared side-by-side, the sensitivity of the optimal investment strategy in terms of relative shares of R\&D investment on both R\&D efficiency and knowledge stock strength is similar in that for the technologies with high learning rates and early period $R \& D$ investments, $R \& D$ investment tends to decrease (or stay constant) with increasing values for both innovation process components. On the other hand, for technologies with low learning rates (e.g., nuclear) and deferred R\&D investments, $R \& D$ investment tends to increase with increasing values of the innovation components. One noteworthy difference in the sensitivities however, as Figure 9 below shows, is that the impact of knowledge stock strength is large enough to change the priority ordering of R\&D investments under a carbon limit. At low levels, the model shows us that it is optimal to invest the majority of the R\&D expenditures on wind, whereas at high levels on nuclear. Given the limited empirical data on these parameters, and the inherent uncertain nature of the innovation process, we leave the exploration of the impact of uncertainty in these parameters as future work. 


\section{Discussion}

In this paper, we present a novel modeling framework for informing investment decisions about $R \& D$ and generation capital infrastructure in the electric power sector. Specifically, the new framework improves upon state-of-the-art modeling in this area by using an explicit engineering-cost structure for representing important power system details such as temporal load variability, technology-specific operational constraints, intermittent renewable generation, and technology-specific engineering and fuel costs. We also utilize a non-linear innovation possibilities frontier function for representing a process of technology knowledge building that captures diminishing returns to R\&D. Results from the model inform decision makers about the relative balance of low-carbon R\&D investments and new capital investments that is cost-effective at transforming the currently high-carbon emitting power generation sector.

In particular, for capital investments, the most competitive emerging technologies (e.g., wind power) are deployed early with and without carbon limits, to meet increasing demand. Without any limits on carbon, the remaining deployment of new capacity is made up of natural gas plants, whereas with a carbon limit, solar PV deployment increases and nuclear power is also deployed. The deployment of nuclear in the presence of a carbon limit is critical — the baseload nature of the technology affords deep emission cuts. R\&D investments generally track the deployment patterns. R\&D investments in early periods are optimal for technologies with high learning rates and do less overall to contribute to emission cuts (e.g., both wind and solar are intermittent generating technologies). However, a greater share of $R \& D$ investment is dedicated to wind than solar, because solar is more resource constrained in the model. Lower initial levels of 
R\&D that increase gradually over the planning horizon are optimal for technologies with lower learning rates and deferred deployment (e.g., nuclear).

The results above demonstrate the value of incorporating critical power sector details and diminishing returns to energy $R \& D$ into the state of the art modeling frameworks. If key characteristics of the electricity sector such as load variability, intermittent generation, and operational constraints such as cycling are omitted, the optimal solutions of the models differ significantly—observable at even the minimumlevel of detail we integrate. Specifically, under a carbon limit, the optimal strategy involves R\&D and capital investments in coal with CCS (whereas in the reference model such investments are not optimal), and the relative share of R\&D investment allocated to coal with CCS grows over time to dominate all other emerging technologies. Likewise, the optimal strategy excludes any investments in solar PV technology. We conclude that the engineering cost structure of the model we use is critical to representing features of the power system such as the ineffectiveness for nuclear plants to cycle and the temporal variability of solar PV generation. If diminishing returns to research effort are not explicitly represented in the model of the innovation process, there is a tendency for the optimal strategy to exhibit underinvestment in future periods. This results from the fact that the average return rate from $R \& D$ investments appears to be much higher in a model without diminishing returns, thus reducing the benefit of continued investments.

A number of questions are raised for future research. First, given the sensitivity of the share of optimal R\&D investments to the components of the knowledge building process such as knowledge stock strength, and the paucity of data available about the actual value of these innovation process components, it would be valuable to study the 
optimal R\&D investment strategy under uncertainty in the return rate of $R \& D$ programs (R\&D efficiency) and knowledge stock strengths. We present one such a study in a forthcoming paper that uses a stochastic R\&D model for the power generation sector, but in general this is an open area of research that would benefit from multiple perspectives. Second, we have developed the current model using an engineering cost structure with the intention of presenting a framework that could be used as the "bottom-up" component of a more integrated hybrid modeling effort. There are many feedbacks between the electricity sector and the rest of the economy, as well as between the energy innovation that occurs in electricity and the rest of the economy, that would be beneficial to include in a modeling framework used to inform investment decisions and related policies. The integration of a modeling framework such as the one presented here into a hybrid model with macroeconomic interactions would be a valuable line of research. Finally, due to our research objective, we have used a stylized model that relies on a single, aggregated entity that engages in innovative activity and chooses R\&D investments only in response to opportunities for minimizing system-wide costs. However, in reality, innovation occurs via several different institutions, through both public and private entities that respond to different incentives. Representing public and private innovation separately in a model like the one we present here could help inform the allocation of R\&D funds across different actors, as would characterizing and representing other key features of the innovation process such as R\&D feedbacks upstream at the technology suppliers from downstream deployment at the utilities and power producers. 


\section{Acknowledgements}

We wish to thank Jake Whitcomb for his excellent research assistance in helping prepare the manuscript for publication. We also thank Andres Ramos, Javier Garcia, and Joseph Sussman for valuable discussions on electricity generation expansion planning and energy innovation, and two anonymous reviewers. Finally, we gratefully acknowledge financial support from U.S. National Science Foundation Grant Nos. 0825915 and 0835414, and the MIT Science and Technology Initiatives Spain Program, Martin Family Sustainability Fellows Program, and Office of the Graduate Dean for Education John S. Hennessy Fellowship Program.

\section{References}

Berglund, C. and P. Soderholm (2006). "Modeling technical change in energy system analysis: analyzing the introduction of learning-by-doing in bottom-up energy models." Energy Policy, 34: 1344-1356.

Blanford, G., Merrick, J., and D. Young (2014). "A Clean Energy Standard analysis with the US-REGEN model." The Energy Journal 35(Special Issue 2): 137-164.

Bosetti, V., Carraro, C., Galeotti, M., Massetti, E., and M. Tavoni (2006). "WITCH A world induced technical change hybrid model." The Energy Journal, Special Issue on Hybrid Modeling of Energy-Environment Policies: Reconciling Bottom-up and Topdown.

Buonanno, P., Carraro, C., and M. Galeotti (2003). "Endogenous induced technical change and the costs of Kyoto." Resource and Energy Economics, 25: 11-34.

Clarke, L., Weyent, J., and A. Birky (2006). "On the sources of technological change: assessing the evidence." Energy Economics 28: 579-595.

DOE (2011). Report on the First Quadrennial Technology Review. DOE. Washington, D.C.

EIA (2012). U.S. Energy-Related Carbon Dioxide Emissions, 2011. DOE/EIA. Washington, D.C.

EIA (2011). Annual Energy Review. DOE/EIA. Washington, D.C.

EIA (2010a). Electric Power Annual 2010. DOE/EIA. Washington, D.C.

EIA (2010b). Updated Capital Cost Estimates for Electricity Generation Plants.

DOE/EIA. Washington, D.C. 
EIA (2009). The National Energy Modeling System: An Overview 2009. DOE/EIA. Washington, D.C.

EPA (2010). Documentation for EPA Base Case v.4.10 Using the Integrated Planning Model. EPA Report \#430R10010. USEPA/Office of Air and Radiation. Washington, D.C.

Fischer, C. and R. G. Newell (2008). "Environmental and technology policies for climate mitigation." Energy Economics, 55: 142-162.

Goulder, L., and K. Mathai (2000). "Optimal $\mathrm{CO}_{2}$ abatement in the presence of induced technological change." Journal of Environmental Economics and Management, 39 (1): $1-38$.

Goulder, L., and S. Schneider (1999). "Induced technological change and the attractiveness of $\mathrm{CO}_{2}$ abatement policies." Resource and Energy Economics, 21: 211253.

Grubb, M., Kohler, J., and D. Anderson (2002). "Induced technical change in energy and environmental modeling; analytic approaches and policy implications." Annu. Rev. Energy Environ, 27: 271-308.

Hobbs, B.F. (1995). "Optimization methods for electric utility resource planning," European Journal of Operational Research, 83: 1-20.

Ibenholt, K. (2002). "Explaining learning curves for wind power." Energy Policy, 30: 1181-1189.

Jones, C. (1995). "R\&D based models of economic growth." The Journal of Political Economy, 103 (4): 759-784.

Klaassen, G., Miketa, A., Larsen, K., and T. Sundqvist (2005). "The impact of R\&D on innovation for wind energy in Demark, Germany and the United Kingdom." Ecological Economics, 54: 227-240.

Kouvaritakis, N., Soria, A., and S. Isoard (2000). "Modelling energy technology dynamics: methodology for adaptive expectations modeling with learning by doing and learning by searching." Int. J. Global Energy Issues, 14 (1-4): 104: 115.

Kypreos, S., and L. Barreto (2000). "ERIS: a model prototype with endogenous technological change." Int. J. Global Energy Issues, 14 (1-4): 374-396.

Loulou, R., Goldstein, G., and K. Noble (2004). Documentation for the MARKAL family of models. IEA/Energy Technology Systems Analysis Programme. Paris. 
Manne, A.S., Mendelsohn, R., and R.G. Richels (1995). "A model for evaluating regional and global effects of GHG reduction policies." Energy Policy, 23: 17-34.

Mattsson, N., and C.O. Wene (1997). "Assessing new energy technologies using an energy system model with endogenized experience curves." International Journal of Energy Research, 21 (4): 385-393.

Messner, S. (1997). "Endogenized technological learning in an energy systems model." $J$ Evol Econ, 7: 291-313.

Miketa, A., and L. Schrattenholzer (2004). "Experiments with a methodology to model the role of R\&D expenditures in energy technology learning processes; first results." Energy Policy, 32: 1679-1692.

Morris, S.C., Goldstein, G.A., and V.M. Fthenakis (2002). "NEMS and MARKALMACRO models for energy-environmental-economic analysis: a comparison of the electricity and carbon reduction projections." Environmental Modeling and Assessment, 7: 207-216.

Nordhaus, W. (2014). "The perils of the learning model for modeling endogenous technological change." The Energy Journal 25(1): 1-14.

Nordhaus, W.D. (2010). "Economic aspects of global warming in a post-Copenhagen environment." Proceedings of the National Academy of Sciences, 107 (26): 11721-11726.

Nordhaus, W.D. (1994). Managing the Global Commons: The Economics of the Greenhouse Effect. Cambridge: MIT Press.

Tapia-Ahumada, K., Octaviano, C., Rausch, S., and I. Pérez-Arriaga (2014). Modeling intermittent renewable energy: can we trust top-down equilibrium approaches? CEEPR Working Paper. MIT. Cambridge.

Palmintier, B. (2013). Incorporating Operational Flexibility into Electric Generation Planning: Impacts and Methods for System Design and Policy Analysis. MIT Engineering Systems Division PhD Dissertation.

Paltsev, S., Reilly, J.M., Jacoby, H.D., Eckaus, R.S., McFarland, J., Sarofim, M., Asadoorian, M., and M. Babiker (2005). The MIT Emissions Prediction and Policy Analysis (EPPA) Model: Version 4, MIT Joint Program on the Science and Policy of Global Change Report Series Report No. 125. Massachusetts Institute of Technology. Cambridge.

Pérez-Arriaga, I.J., and C. Meseguer (1997). "Wholesale marginal prices in competitive generation markets." IEEE Transactions on Power Systems, 12 (2): 710-717. 
Popp, D. (2002). "Induced innovation and energy prices." American Economic Review, 92 (1): $160-180$.

Popp, D. (2004). "ENTICE: endogenous technological change in the DICE model of global warming." Journal of Environmental Economics and Management, 48: 742-768.

Popp, D. (2006). "ENTICE-BR: Backstop technology in the ENTICE model of climate change." Energy Economics, 28(2): 188-222.

Popp, D., Newell, R.G., and A.B. Jaffe (2010). "Energy, the environment, and technological change," Handbook of the Economics of Innovation: vol. 2, Bronwyn Hall and Nathan Rosenberg, eds., Academic Press/Elsevier, 2010, 873-937.

Popp, D., Santen, N., Fisher-Vanden, K., and M. Webster (2013). “Technology Variation vs. R\&D Uncertainty: What Matters Most for Energy Patent Success?" Resource and Energy Economics, 35: 505-533.

Graham P., Clarke, L., Marlay, R., Kyle, P., Wise, M., McJeon, H., and G. Chan (2011). "Energy R\&D portfolio analysis based on climate change mitigation." Energy Economics, 33 (4): 634-643.

Ross, M. (2008). Documentation of the applied dynamic analysis of the global economy (ADAGE) model. Research Triangle Institute Working Paper 08_01. RTI. Research Triangle Park.

Rubin, E.S., Taylor, M.R., Yeh, S., and D.A. Hounshell (2004). "Learning curves for environmental technology and their importance for climate policy analysis." Energy, 29: 1551-1559.

Seebregts. A.J., Kram, T., Schaeffer, G.J., Stoffer, A., Kypreos, S., Barreto, L., Messner, S., and L. Schrattenholzer (1999). Endogenous technological change in energy system models, synthesis of experience with ERIS, MARKAL, and MESSAGE. Report ECNC - 99-025 of the Paul Scherrer Institute and the International Institute for Applied Systems Analysis. PSI/IIASA. Switzerland/Austria.

Shawhan, D.L., Taber, J.T., Shi, D., Zimmerman, R.D., Yan, J., Marquet, C.M., Qi, Y., Mao, B., Schuler, R.E., Schulze, W.D., and D. Tylavsky (2014). "Does a detailed model of the electricity grid matter? Estimating the impacts of the Regional Greenhouse Gas Initiative." Resource and Energy Economics 36: 191-207.

Short, W., Sullivan, P., Mai, T., Mowers, M., Uriate, C., Blair, N., Heimiller, D., and A. Martinez (2011). Regional Energy Deployment System (ReEDS). Technical Report NREL/TP-6A20-46534. NREL. Golden.

Short, W., Blair, N., Sullivan, P., T. Mai (2009). ReEDS model documentation: base case data and model description. NREL. Golden. 
Soderholm, P. and G. Klaassen (2007). "Wind power in Europe: a simultaneous innovation-diffusion model." Environmental and Resource Economics, 36: 163-190.

Turton, H., and L. Barreto (2004). The extended energy-systems ERIS model: an overview. International Institute for Applied Systems Analysis, Interim Report IR-04101. IIASA. Vienna.

Turvey, R., and D. Anderson (1977). Electricity Economics: Essays and Case Studies. Baltimore: Johns Hopkins University Press.

van der Zwaan, B., Gerlagh, R., Klaassen, G., and L. Schrattenholzer (2002).

"Endogenous technological change in climate change modeling." Energy Economics, 24: $1-19$. 
Table 1. Electricity Generator Data

\begin{tabular}{|c|c|c|c|c|c|c|c|c|c|}
\hline Technology & $\begin{array}{c}\text { Initial } \\
\text { Capacity } \\
{[\mathrm{GW}]}\end{array}$ & $\begin{array}{c}\text { 5-year } \\
\text { Retirement } \\
\text { Rate }[\%]\end{array}$ & $\begin{array}{c}\text { Heat Rate } \\
{[\mathrm{MMbtu} / \mathrm{MWh}]}\end{array}$ & $\begin{array}{c}\text { Initial Capital } \\
\text { Cost }[\$ / \mathrm{kW}- \\
\text { knowledgeunit] }\end{array}$ & $\begin{array}{c}\text { Fixed } \\
\text { O\&M } \\
\text { Cost } \\
\text { [\$/kW- } \\
\text { year] }\end{array}$ & $\begin{array}{c}\text { Initial } \\
\text { Fuel Cost } \\
{[\$ / \mathrm{MMBtu}]}\end{array}$ & $\begin{array}{c}\text { Other } \\
\text { Variable } \\
\text { Cost } \\
\text { [\$/MWh] }\end{array}$ & $\begin{array}{c}\text { Emissions } \\
\text { Rate } \\
{[\mathrm{lbs} / \mathrm{MMbtu}]}\end{array}$ & $\begin{array}{c}\text { Annual } \\
\text { Availability } \\
\text { Rate }[\%]\end{array}$ \\
\hline Old Coal & 300 & 15 & 10.00 & 1204 & 23.410 & 2.28 & 4.14 & 205 & 85 \\
\hline New Coal & 1 & - & 8.80 & 3167 & 35.970 & 2.28 & 4.25 & 205 & 85 \\
\hline $\begin{array}{l}\text { Coal with } \\
\text { CCS }\end{array}$ & 1 & - & 12.00 & 5099 & 76.620 & 2.28 & 9.05 & 20.5 & 80 \\
\hline $\begin{array}{l}\text { Old Steam } \\
\text { Gas } \\
\text { Gas }\end{array}$ & 100 & 20 & 9.46 & 390 & 25.256 & 5.16 & 3.85 & 137 & 80 \\
\hline $\begin{array}{c}\text { Combined } \\
\text { Cycle } \\
\text { Gas }\end{array}$ & 200 & - & 6.43 & 1003 & 14.620 & 5.16 & 3.11 & 119 & 90 \\
\hline $\begin{array}{l}\text { Combustion } \\
\text { Turbine }\end{array}$ & 100 & - & 9.75 & 665 & 6.700 & 5.16 & 9.87 & 119 & 90 \\
\hline Hydro & 100 & - & 10.34 & 1320 & 12.700 & - & 3.20 & - & 90 \\
\hline Nuclear & 100 & 10 & 10.40 & 5355 & 85.663 & 0.62 & 0.48 & - & 90 \\
\hline Wind & 50 & - & - & 2438 & 28.070 & - & - & - & 30 \\
\hline Solar & 1 & - & - & 4755 & 16.700 & - & - & - & $95 * *$ \\
\hline
\end{tabular}

Notes:

** The availability rate for solar is high due to the technology only operating during peak solar demand slices.

References: Short et al., 2011; EIA, 2010a; EIA, 2010b

\section{Table 2. Other Used Parameters}

\begin{tabular}{lc}
\multicolumn{1}{c}{ Parameter } & Value \\
\hline Cumulative Emissions Cap No Policy/Policy, ecap & $140,000 / 60,000$ Million Metric Tons \\
Electricity Supply Reliability Reserve Margin, $m$ & $10 \%$ \\
Annual Electricity Demand Growth Rate, $k$ & $0.5 \%$ \\
Annual Discount Rate, $r$ & $5 \%$ \\
Coal Price Annual Growth Rate, fuel_growth & $4 \%$ \\
Gas Price Annual Growth Rate, fuel_growt & \\
\end{tabular}




\begin{tabular}{|c|c|c|c|c|c|}
\hline Technology & $\begin{array}{c}\text { Learning-by- } \\
\text { Doing Elasticity } \eta 1\end{array}$ & $\begin{array}{c}\text { Learning-by- } \\
\text { Searching } \\
\text { Elasticity } \eta 2\end{array}$ & IPF a & IPF $\beta$ & IPF $\phi$ \\
\hline Coal with $C{ }^{x i}$ & 0.05889 & 0.02915 & 0.1853 & 0.1 & 0.54 \\
\hline Nuclear & 0.05889 & 0.02915 & 0.1853 & 0.1 & 0.54 \\
\hline Wind & 0.25154 & 0.10470 & 0.1856 & 0.1 & 0.54 \\
\hline Solar & 0.41504 & 0.15200 & 0.1760 & 0.1 & 0.54 \\
\hline
\end{tabular}

Original Sources: Barreto \& Kypreos 2004; Popp 2006

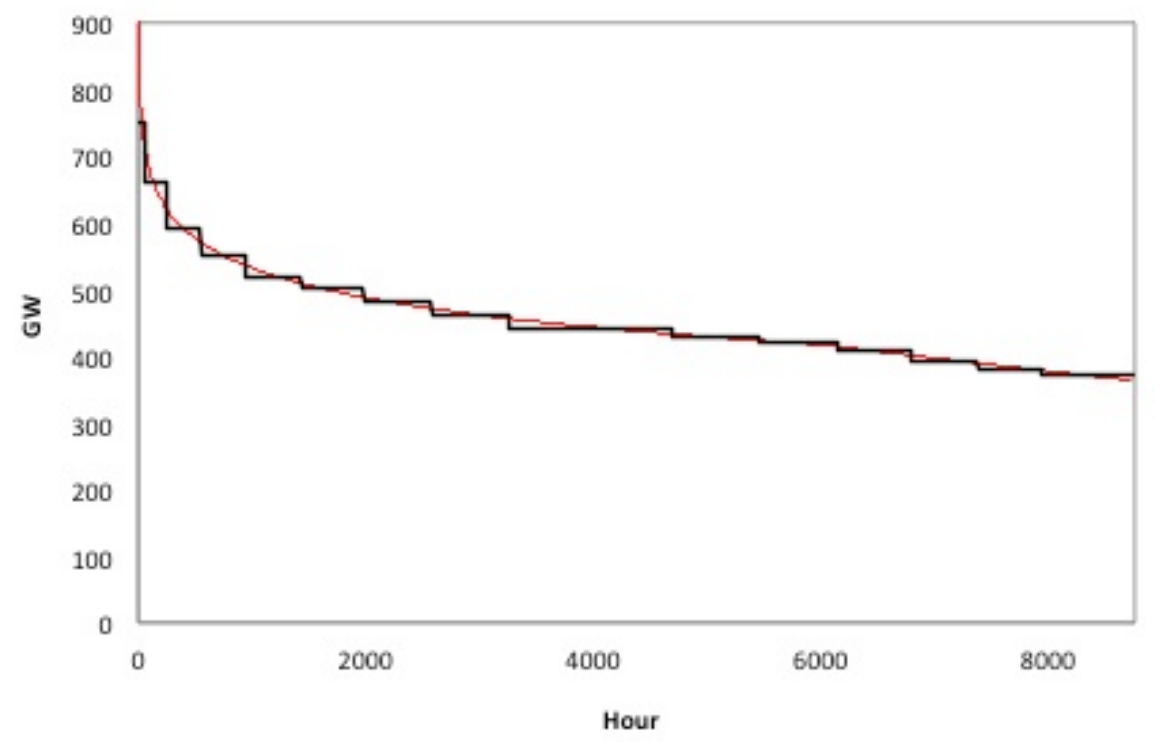

Figure 1. Load Duration Curve Used (Original Source: Short et al. 2009)

\footnotetext{
${ }^{x i}$ The lack of experience with carbon capture and sequestration technology in the electric power sector makes it difficult to find reliable learning data for use in numerical models of technological change. Thus, other authors have used learning rates for coal $\mathrm{SO}_{2}$ scrubbing technology or $\mathrm{NO}_{\mathrm{x}}$ reduction technologies and applied them to coal with CCS technology in numerical decision support models (Rubin, Taylor, Yeh, $\&$ Hounshell 2004). This paper uses the history of nuclear fission technology and its learning rates as a proxy for coal with CCS (both are capital-intensive, large baseload technologies with significant challenges of space, scale up, public acceptance, permitting, waste, etc.).
} 

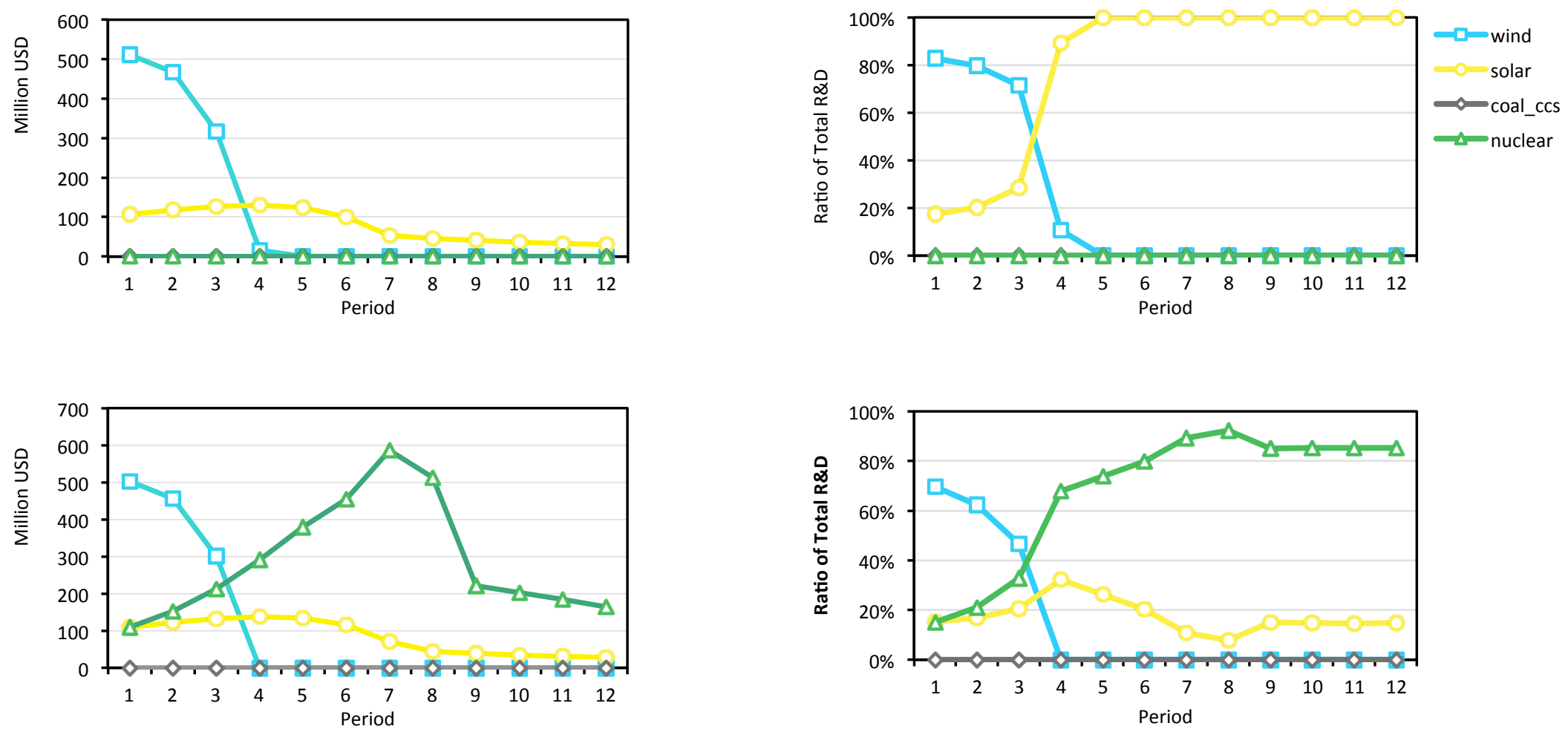

Figure 2. Left: Reference model optimal R\&D investments under no carbon limit (top) and a carbon limit (bottom). Right: Ratio of optimal R\&D investments to total R\&D investments per period, for the reference model under no carbon limit (top) and a carbon limit (bottom). 

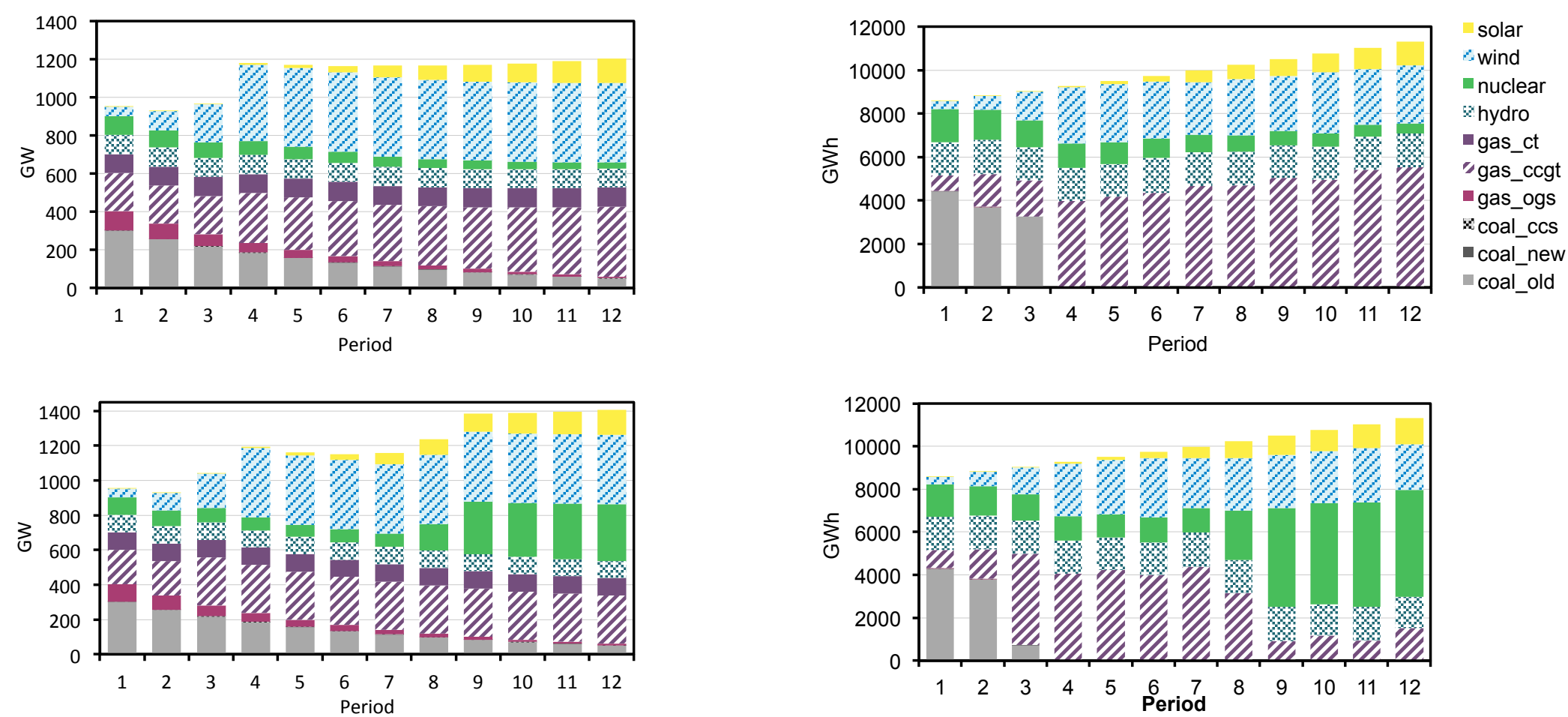

Figure 3. Left: Reference model optimal installed generation capacity under no carbon limit (top) and a carbon limit (bottom). Right: Reference model optimal electricity production under no carbon limit (top) and a carbon limit (bottom). 

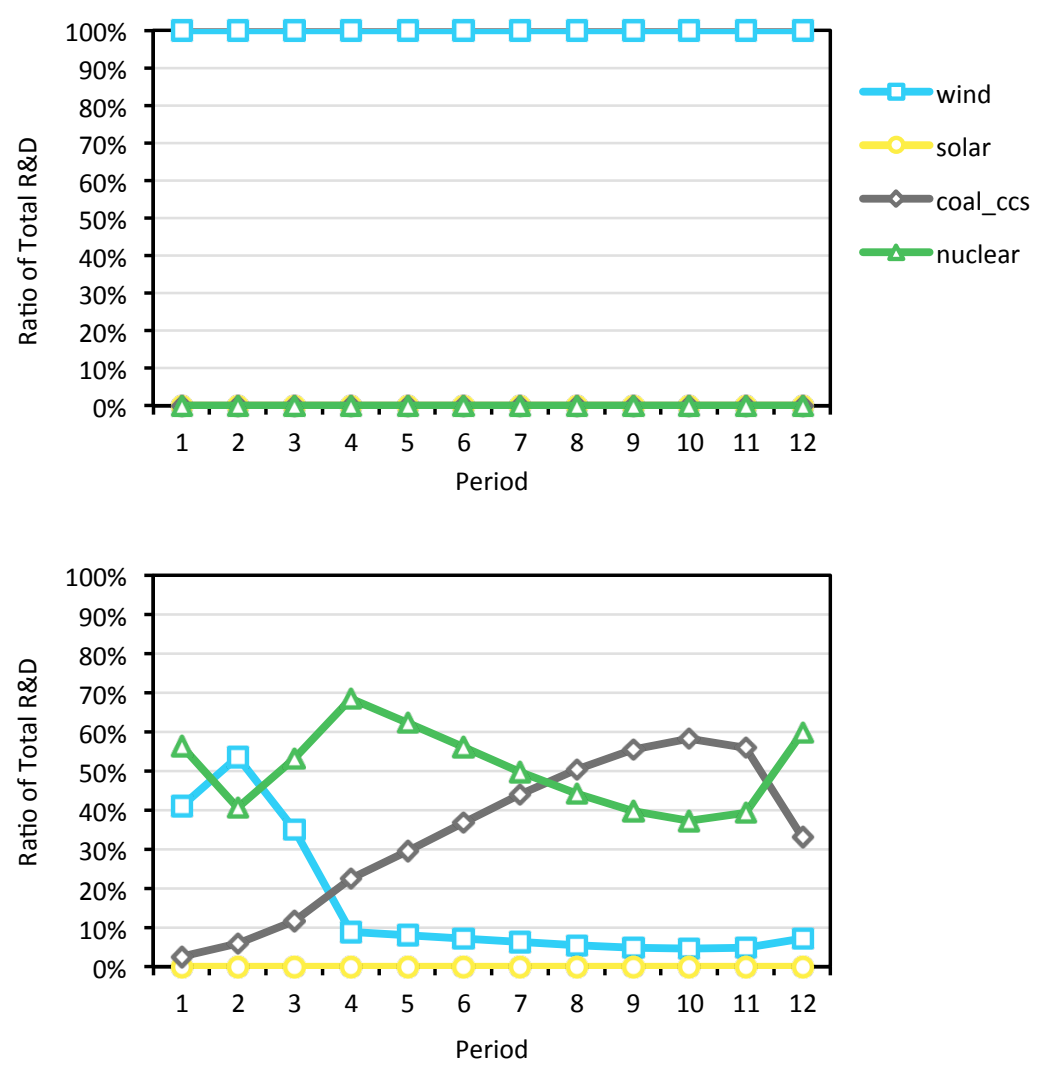

Figure 4. Ratio of optimal R\&D investments to total R\&D investments per period, without power system details modeled, under no carbon limit (top) and a carbon limit (bottom). 

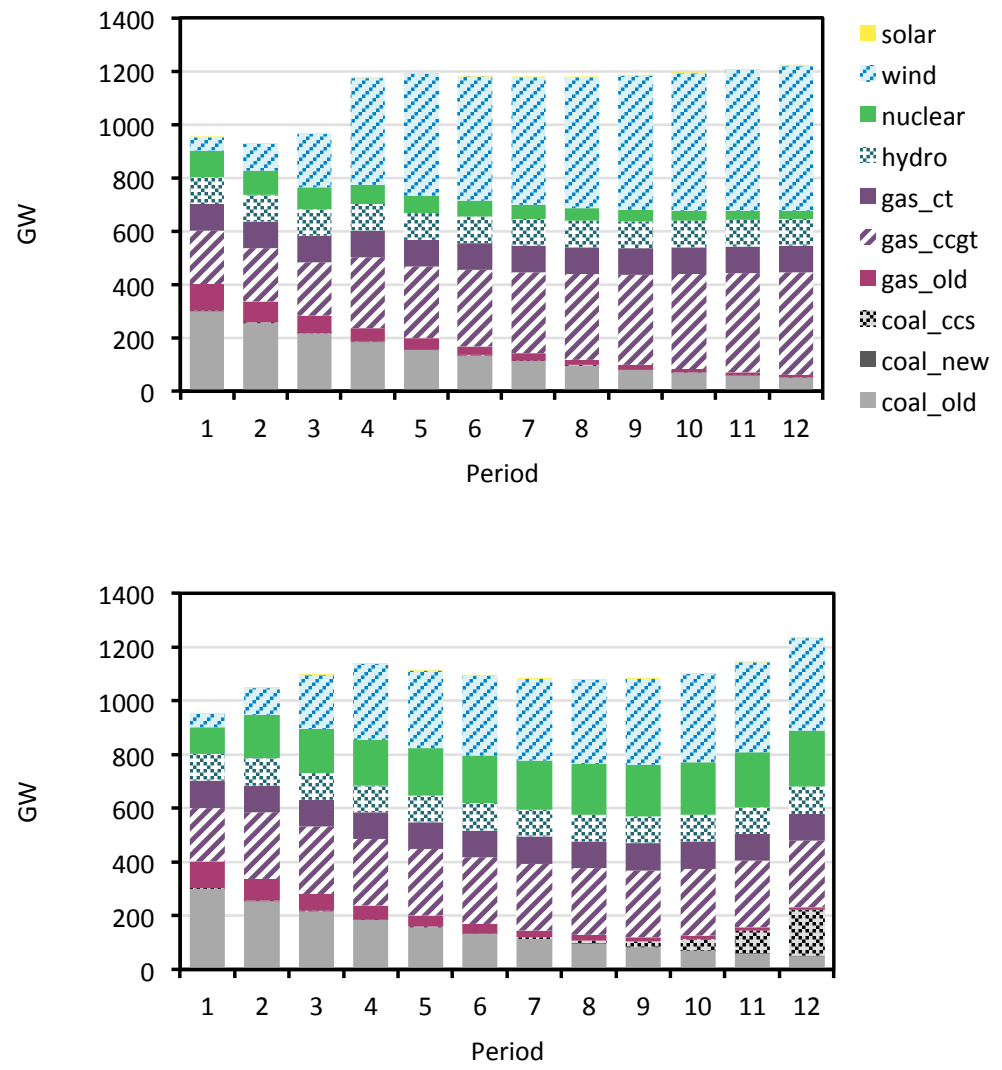

Figure 5. Optimal installed capacities without power system details modeled, under no carbon limit (top) and a carbon limit (bottom). 

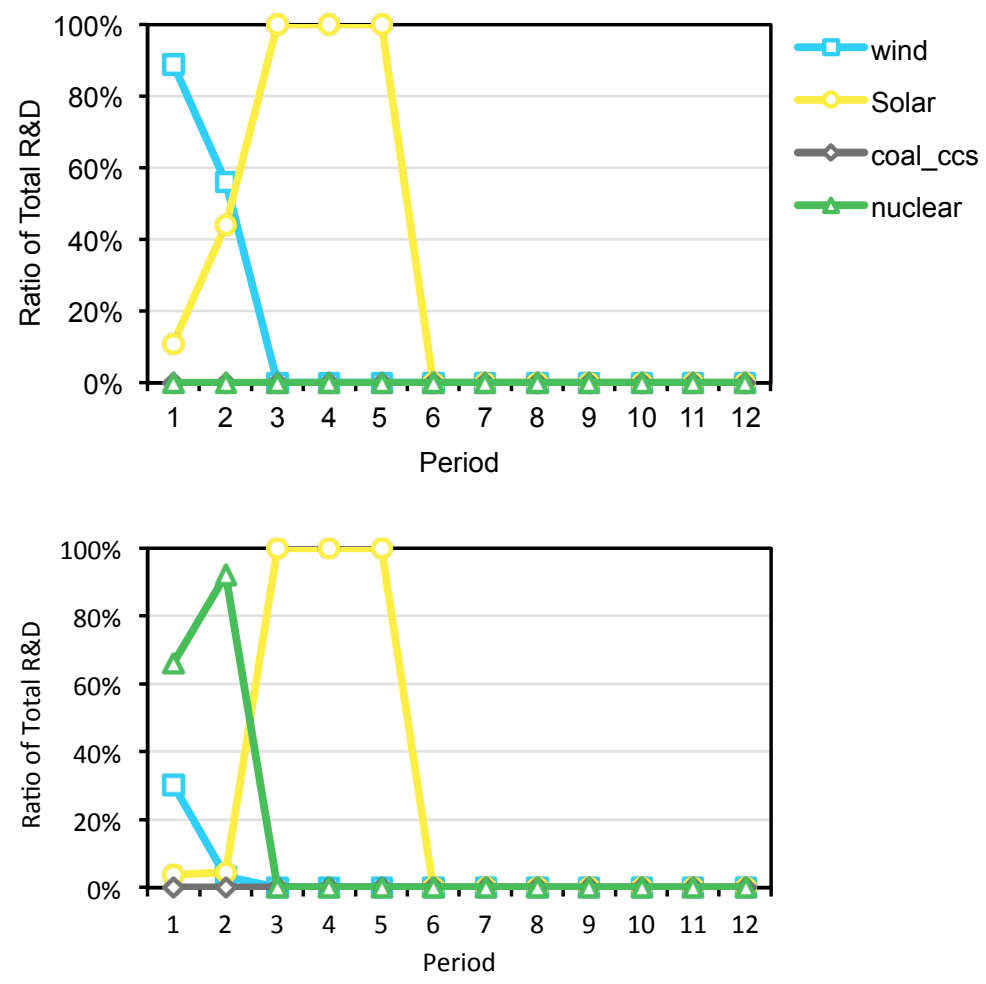

Figure 6. Optimal R\&D investments without diminishing returns to research, under no carbon limit (top) and under a carbon limit (bottom).

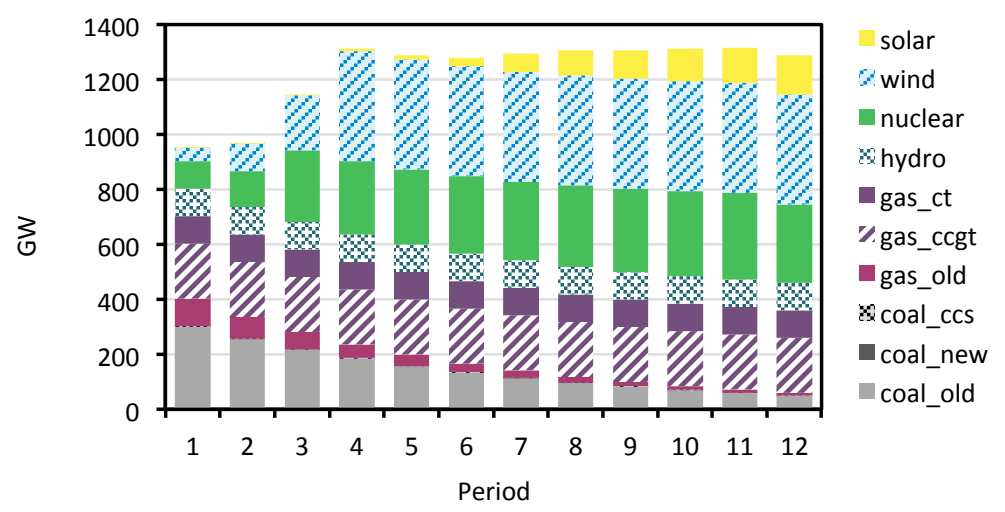

Figure 7. Optimal installed capacities without diminishing returns to research, under a carbon limit. 


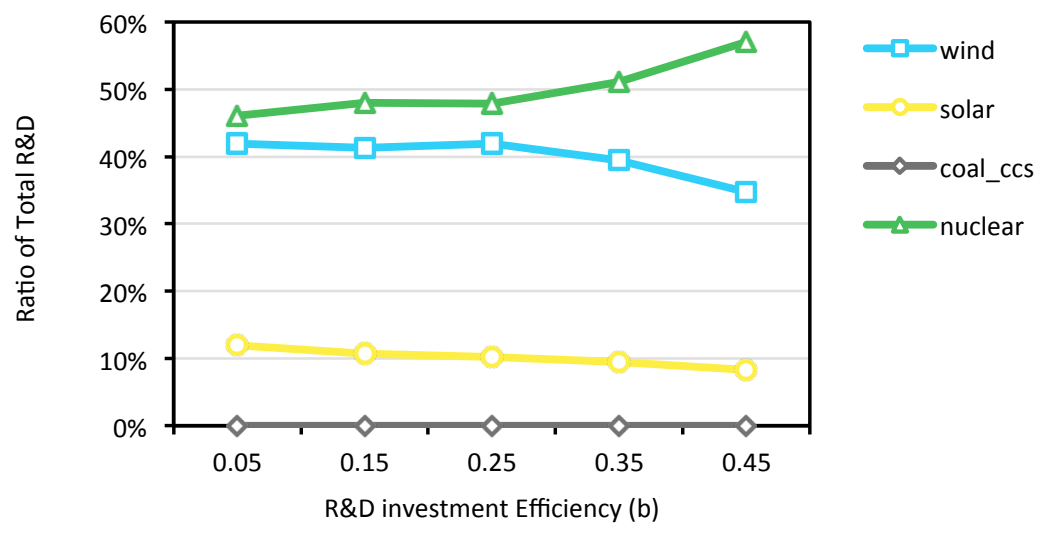

Figure 8. Peak optimal $R \& D$ investments for various $R \& D$ efficiencies (value for $\beta$ in the Innovation Possibilities Frontier) under a carbon limit.

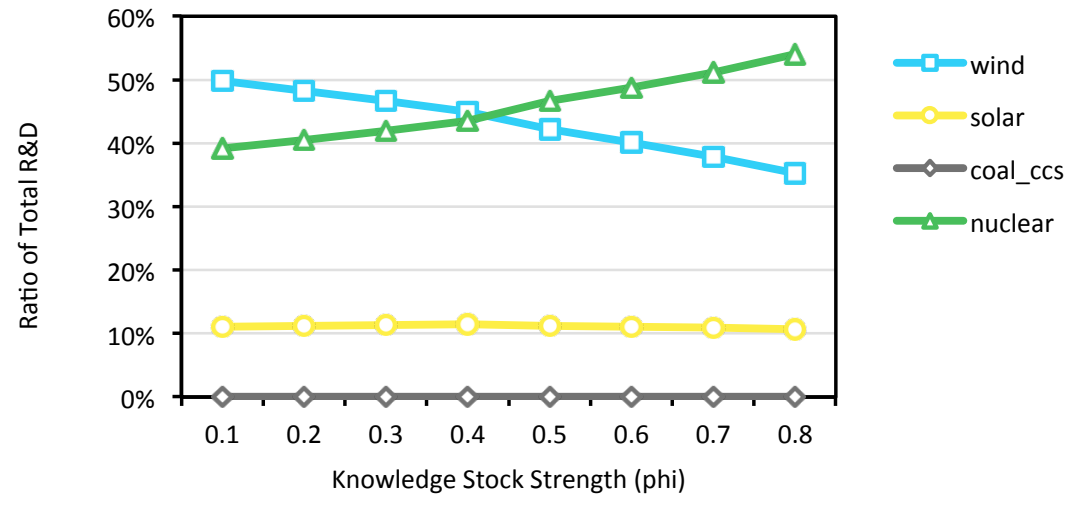

Figure 9. Peak optimal R\&D investments for various knowledge stock strengths ( $\varphi$ values in the "Innovation Possibilities Frontier") under a carbon limit. 